BIOKEMISTRI 20(1):1-9 (June 2008)

This article is downloadable online in PDF format

at http://www.bioline.org. $\mathrm{br} / \mathrm{bk}$

Printed in Nigeria
An international journal published by the

? त्रिके

OVigerian Society for O̊xperimental \&iology

\title{
Effect of heat processing on the proximate composition and energy values of selected Nigerian staple foods from oil-producing areas of the Niger Delta
}

\author{
Eugene N. ONYEIKE ${ }^{1}$, Joyce O. AKANINWOR ${ }^{1}$ and Jonathan C. IFEMEJE ${ }^{2}$ \\ ${ }^{1}$ Department of Biochemistry, Faculty of Science, University of Port Harcourt, PMB 5323, Port Harcourt, \\ Nigeria \\ ${ }^{2}$ Department of Biochemistry, Faculty of Science, Anambra State University, PMB 02, Uli, Nigeria
}

Received 5 August 2006

MS/No BKM/2006/027, (c) 2008 Nigerian Society for Experimental Biology. All rights reserved.

\begin{abstract}
Proximate composition and energy values of four raw and heat processed staple foods (yams, cassava, cocoyam and maize) from oil-producing (Eleme, Ogoni, Okrika, and Nembe) areas of Rivers and Bayelsa States and Abakaliki (a non-oil producing area in Ebonyi State, Nigeria) as control were investigated. In raw yam tubers, moisture was high in all the locations and ranged from $61.96 \pm 0.02 \%$ in Abakaliki to $63.77 \pm 0.25 \%$ in Ogoni. Crude protein ranged from $1.39 \pm 0.13 \%$ in Okrika to $1.71 \pm 0.06 \%$ in Abakaliki while ash was highest $(2.57 \pm 0.12 \%)$ in Okrika followed by $2.33 \pm 0.01 \%$ in Eleme and was lowest $(0.97 \pm 0.06 \%)$ in Abakaliki. Crude fat was low in all the locations and ranged from $0.10 \pm 0.00 \%$ in Okrika to $0.17 \pm 0.01 \%$ in Nembe while total carbohydrate was high in all the locations ranging from $33.32 \pm 0.42 \%$ in Ogoni to $35.21 \pm 0.13 \%$ in Abakaliki. The calorific value (kcal/100 g sample) in all the locations was low. It was highest (149.08) in yams from Abakaliki followed by Eleme (142.55) and lowest in Ogoni (140.09). Similar trends in values of the constituents were obtained in raw cassava, cocoyam and maize from different oil-producing and control locations investigated. In each of these locations, heat processing generally increased moisture content of all the samples (yam, cassava products, cocoyam and maize), but decreased dry matter, crude protein, ash, crude fat, total carbohydrate and calorific value. With the exception of moisture and ash, other nutrients were significantly $(\mathrm{p}<0.05)$ higher in the control area of Abakaliki than values obtained in similar samples from oil producing areas of the Niger Delta investigated
\end{abstract}

Keywords: Nigerian staple foods, Niger Delta, heat processing, proximate composition, energy values

*To whom correspondence should be addressed. E-mail: enonyeike@yahoo.com Tel: 0803-6714403 


\section{INTRODUCTION}

In Nigeria, there are 36 States and the Federal Capital Territory, Abuja divided into six geopolitical zones (Northeast, Northwest, Northcentral, Southeast, Southwest and South south) Rivers and Bayelsa in the Southsouth zone are two of the states in oil-producing areas of the Niger Delta, Nigeria where staple foods such as yam, cassava, cocoyam and maize are abundant, though the staples are seasonal. Oilproducing areas (Eleme, Ogoni, Okrika and Nembe) are fraught with petroleum exploration and exploitation and these have led to wide scale pollution of its farmlands (soils), rivers and underground water with hydrocarbons and dispersant products ${ }^{1}$.

Crude oil spillages on soil and indiscriminate discharge of effluents on farmlands contaminate the soils and water bed making them polluted and unsatisfactory for agricultural purposes ${ }^{2}$. Crude oil spillage exerts adverse effect on plants by making toxic minerals in the soil available to plants and by reducing the soil $\mathrm{pH}$; when soil $\mathrm{pH}$ drops below 4.50 , the productivity of most crops are adversely affected ${ }^{3,4}$. As the people of Rivers and Bayelsa States are predominantly subsistent farmers, the bulk of plant nutrients (carbohydrate, fats, proteins, ash, moisture and fibre) in their diets are derived from the consumption of staple foods, (starchy root tubers, cereals and legumes).

Foods are required by humans for growth, development, reproduction and energy. The poor nutritional status of Nigerians and malnutritional disorders are as a result of complex factors such as pollution, low productivity and quality and the presence of toxicants in foods which need to be destroyed by heat treatment. For instance, heat treatment has been shown to inactivate trypsin inhibitors from the African yam bean ${ }^{5}$.

Yams are species of Dioscorea and belong to Dioscoreacea family propagated from tubers and grow best in deep well drained soils. They are pealed, boiled and pounded to produce a gelatinous dough called pounded yam which is consumed with soup. Yam flour as a local soup thickener is used to prepare afiaefere or white soup $^{6}$, and is a good source of $\operatorname{starch}^{6,7}$. Cassava (Manihot esculenta) is a root crop that belongs to the family Euphorbiacea and is propagated from stem cuttings. The root is a cheap source of carbohydrate for human and livestock.

Fermented cassava roots are boiled and pounded into a thick paste called fufu which is consumed with soup. The root can be cooked and eaten with red palm oil and sauces. The roots may be peeled, grated, fermented and fried with or without red palm oil into garri flour in which form it is mainly consumed in Nigeria ${ }^{6}$. Taro cocoyam (Colocasia esculenta) and tannia species (Xanthosoma saggitifolium) are mostly available and consumed members of the Aracaea family. Young leaves of taro cocoyam are eaten in Nigeria as in other West African countries as vegetable food for ruminant particularly sheep and goat ${ }^{7}$. Cocoyam is a good source of carbohydrate and energy and is usually eaten boiled or roasted.

Maize is the second most import cereal crop in Nigeria ranking close to sorghum in the number of people it feeds. The grains are eaten roasted or boiled. They can be milled to produce flour used in the preparation of dishes such as pap. These staple food materials are of important nutritional value. The present work was carried out to determine the proximate composition of staple foods produced from oil-producing areas of the Niger Delta, and the effect of heat processing on their nutrient status.

\section{MATERIALS AND METHODS}

\section{Samples}

The samples were yam (Dioscorea rotundata), cassava (Manihot esculenta), cocoyam (Colocasia esculenta) and maize (Zea mays) which were freshly harvested from the study areas (Eleme, Ogoni, Okrika, Nembe) and the control (Abakaliki in Ebonyi State). Garri and Fufu samples were locally processed from cassava.

\section{Sample preparation}

Each of yams, cassava and cocoyam was peeled to remove the coat, sliced and ground into slurry to obtain raw sample. 


\section{Sample Processing}

Each of composite samples of cocoyam was peeled, cooked to tenderness and the cooking solution discarded. They were then oven-dried at $105^{\circ} \mathrm{C}$ for $18 \mathrm{~h}$, ground into flour using a food grinder to pass a $75 \mu \mathrm{m}$ mesh size and stored in a clean dry air-tight container in a refrigerator $\left(4^{0} \mathrm{C}\right)$ until required for analyses. The maize grains from the cobs were also cooked to tenderness and processed as above for yams and cocoyam.

\section{Analyses of Samples}

Each of composite samples was pooled and proximate analysis was carried out to determine moisture, crude protein, ash, crude fat and total carbohydrate. The recommended methods of the Association of Official Analytical Chemists ${ }^{8}$ were adopted. The $\mathrm{AOAC}^{8}$ method numbers were $14.004,2.057,14.006$ and 7.062 for moisture, crude protein, ash and crude fat. Moisture content was obtained by heating three $5.0 \mathrm{~g}$ portions of each of the samples to a constant weight in a hot air-circulating oven (Plus II Sanyo, Gallenkamp PLC, England) at $105^{\circ} \mathrm{C}$ for $24 \mathrm{hr}$. Crude protein $(\% \mathrm{~N} \mathrm{x} 6.25)$ was obtained by the Kjeldahl method in which nitrogen was determined and \% Nitrogen multiplied by a factor of 6.25 to obtain \% protein. The method involved the digestion of three $1.0 \mathrm{~g}$ samples with concentrated tetraoxosulphate (VI) acid, $\mathrm{H}_{2} \mathrm{SO}_{4}$, the distillation of the digest to liberate ammonia $\left(\mathrm{NH}_{3}\right)$ which was trapped into $2.0 \%$ boric acid solution. This was followed by titration with
$0.10 \mathrm{M} \mathrm{HCl}$ and titre value used to calculate percent nitrogen.

Ash was determined by the incineration of three $1.0 \mathrm{~g}$ samples in a muffle furnace (LMF4 Carbolite, Bamford, Sheffield, UK) at $650^{\circ} \mathrm{C}$ for 3h. Crude fat was obtained by exhaustively extracting three replicates of $10.0 \mathrm{~g}$ samples in the Soxhlet apparatus using petroleum ether (bp $40-60^{\circ} \mathrm{C}$ ) as the extractant as reported ${ }^{8,9}$. Total carbohydrate was calculated by the difference method (summing the values of moisture, crude protein, ash and crude fat and subtracting the sum from 100) as reported ${ }^{9}$. The calorific value was calculated by multiplying the mean values of crude protein, crude fat and total carbohydrate by the Atwater factors of 4, 9 and 4, respectively, taking the sum of the products and expressing the result in $\mathrm{kcal}^{100 \mathrm{~g}^{-1}}$ sample $^{10}$.

\section{Data Analyses}

Data were analysed statistically by one way analysis of variance (ANOVA) technique. Means were compared by the Duncan's ${ }^{11}$ multiple range test, and significance was accepted at 5\% level ( $\mathrm{p}$-value $=0.05)$.

\section{RESULTS}

The proximate composition of raw and heat processed yam tubers from oil-producing areas of the Niger Delta and Abakaliki (control) are presented in Table 1. In raw samples, moisture was highest in Ogoni $(63.77 \pm 0.25 \%)$ followed by Nembe $(63.50 \pm 0.10 \%)$ and was lowest in Abakaliki (61.96 $\pm 0.02 \%)$.

Table 1: Proximate Composition (\%) of Raw and Heat Processed Yam Tubers from Oil Producing Areas (Eleme, Ogoni, Okrika, Nembe) and Abakaliki

Proximate composition (\%)*

Constituent:

Raw Samples in Location

\begin{tabular}{llllll} 
& Eleme & Ogoni & Okrika & Nembe & Abakaliki \\
\hline Moisture content & $62.18 \pm 0.02^{\mathrm{d}}$ & $63.77 \pm 0.25^{\mathrm{a}}$ & $62.50 \pm 0.12^{\mathrm{c}}$ & $63.50 \pm 0.10^{\mathrm{b}}$ & $61.96 \pm 0.02^{\mathrm{d}}$ \\
Dry matter & $37.82 \pm 0.02^{\mathrm{a}}$ & $36.23 \pm 0.25^{\mathrm{d}}$ & $37.50 \pm 0.12^{\mathrm{b}}$ & $36.50 \pm 0.10^{\mathrm{c}}$ & $38.04 \pm 0.02^{\mathrm{a}}$ \\
Crude protein & $1.57 \pm 0.23^{\mathrm{ab}}$ & $1.42 \pm 0.11^{\mathrm{b}}$ & $1.39 \pm 0.13^{\mathrm{b}}$ & $1.50 \pm 0.17^{\mathrm{b}}$ & $1.71 \pm 0.06^{\mathrm{a}}$ \\
Ash & $2.33 \pm 0.01^{\mathrm{b}}$ & $1.37 \pm 0.12^{\mathrm{c}}$ & $2.57 \pm 0.12^{\mathrm{a}}$ & $1.43 \pm 0.06^{\mathrm{c}}$ & $0.97 \pm 0.06^{\mathrm{d}}$ \\
Crude fat & $0.12 \pm 0.01^{\mathrm{d}}$ & $0.13 \pm 0.01^{\mathrm{c}}$ & $0.10 \pm 0.00^{\mathrm{e}}$ & $0.17 \pm 0.01^{\mathrm{a}}$ & $0.16 \pm 0.01^{\mathrm{b}}$ \\
Total carbohydrate & $33.81 \pm 0.16^{\mathrm{b}}$ & $33.32 \pm 0.42^{\mathrm{b}}$ & $33.45 \pm 0.23^{\mathrm{b}}$ & $33.47 \pm 0.32^{\mathrm{b}}$ & $35.21 \pm 0.13^{\mathrm{a}}$ \\
$\begin{array}{l}\text { Calorific value } \\
\text { (k cal/100g sample) }\end{array}$ & $142.55 \pm 0.35^{\mathrm{b}}$ & $140.09 \pm 1.42^{\mathrm{c}}$ & $140.23 \pm 0.91^{\mathrm{c}}$ & $141.09 \pm 0.59^{\mathrm{b}, \mathrm{c}}$ & $149.08 \pm 0.28^{\mathrm{a}}$
\end{tabular}


Table 1 (contd):Heat Processed Samples in Location

\begin{tabular}{llllll} 
& Eleme & Ogoni & Okrika & Nembe & Abakaliki \\
\hline Moisture content & $66.05 \pm 0.02^{\mathrm{b}}$ & $65.31 \pm 0.02^{\mathrm{c}}$ & $64.01 \pm 0.02^{\mathrm{e}}$ & $64.47 \pm 0.19^{\mathrm{d}}$ & $66.57 \pm 0.01^{\mathrm{a}}$ \\
& & & & & \\
Dry matter & $33.95 \pm 0.02^{\mathrm{d}}$ & $34.69 \pm 0.06^{\mathrm{c}}$ & $35.99 \pm 0.02^{\mathrm{a}}$ & $35.53 \pm 0.02^{\mathrm{b}}$ & $33.43 \pm 0.01^{\mathrm{e}}$ \\
Crude protein & $1.31 \pm 0.22^{\mathrm{a}, \mathrm{b}}$ & $1.25 \pm 0.04^{\mathrm{a}, \mathrm{b}}$ & $1.15 \pm 0.05^{\mathrm{b}, \mathrm{c}}$ & $0.99 \pm 0.52^{\mathrm{c}}$ & $1.39 \pm 0.01^{\mathrm{a}}$ \\
Ash & $1.75 \pm 0.07^{\mathrm{a}}$ & $1.35 \pm 0.15^{\mathrm{b}}$ & $1.85 \pm 0.05^{\mathrm{a}}$ & $1.20 \pm 0.00^{\mathrm{c}}$ & $0.55 \pm 0.04^{\mathrm{d}}$ \\
Crude fat & $0.10 \pm 0.00^{\mathrm{e}}$ & $0.14 \pm 0.01^{\mathrm{b}}$ & $0.12 \pm 0.01^{\mathrm{d}}$ & $0.24 \pm 0.00^{\mathrm{a}}$ & $0.13 \pm 0.01^{\mathrm{c}}$ \\
Total carbohydrate & $30.78 \pm 0.15^{\mathrm{e}}$ & $31.95 \pm 0.15^{\mathrm{c}}$ & $32.87 \pm 0.02^{\mathrm{b}}$ & $33.18 \pm 0.05^{\mathrm{a}}$ & $31.37 \pm 0.05^{\mathrm{d}}$ \\
$\begin{array}{l}\text { Calorific value } \\
\text { (k cal/100 g sample) }\end{array}$ & $129.29 \pm 0.28^{\mathrm{e}}$ & $134.02 \pm 0.39^{\mathrm{c}}$ & $137.12 \pm 0.31^{\mathrm{b}}$ & $138.82 \pm 0.36^{\mathrm{a}}$ & $132.16 \pm 0.22^{\mathrm{d}}$ \\
\hline
\end{tabular}

*Values are means tstandard deviations of triplicate determinations. Values in the same row (for raw and for heat processed) bearing the same superscript letters are not significantly different at 5\% level.

Table 2: Proximate Composition (\%) of Raw and Heat Processed Cassava from Oil Producing Areas (Eleme, Ogoni, Okrika, Nembe) and Abakaliki

\begin{tabular}{|c|c|c|c|c|c|}
\hline \multirow{3}{*}{ Constituents: } & \multicolumn{3}{|c|}{ Proximate Composition (\%)* } & \multirow[b]{3}{*}{ Nembe } & \multirow[b]{3}{*}{ Abakaliki } \\
\hline & \multicolumn{3}{|c|}{ Raw Samples in Location } & & \\
\hline & Eleme & Ogoni & Okrika & & \\
\hline Moisture content & $62.29 \pm 0.02^{\mathrm{c}}$ & $62.95 \pm 0.05^{\mathrm{b}}$ & $63.21 \pm 0.01^{\mathrm{a}}$ & $62.94 \pm 0.14^{\mathrm{b}}$ & $62.10 \pm 0.01^{\mathrm{d}}$ \\
\hline Dry matter & $37.71 \pm 0.02^{\mathrm{b}}$ & $37.05 \pm 0.05^{\mathrm{c}}$ & $36.79 \pm 0.01^{\mathrm{d}}$ & $37.06 \pm 0.14^{\mathrm{c}}$ & $37.90 \pm 0.01^{\mathrm{a}}$ \\
\hline Crude protein & $0.55 \pm 0.15^{\mathrm{a}}$ & $0.50 \pm 0.06^{\mathrm{a}, \mathrm{b}}$ & $0.33 \pm 0.15^{\mathrm{c}}$ & $0.38 \pm 0.06^{\mathrm{bc}}$ & $0.55 \pm 0.11^{\mathrm{a}}$ \\
\hline Ash & $1.63 \pm 0.15^{\mathrm{a}}$ & $0.83 \pm 0.06^{\mathrm{c}}$ & $1.13 \pm 0.06^{\mathrm{b}}$ & $1.80 \pm 0.17^{\mathrm{a}}$ & $0.87 \pm 0.12^{\mathrm{c}}$ \\
\hline Crude fat & $0.20 \pm 0.01^{\mathrm{b}}$ & $0.25 \pm 0.01^{\mathrm{a}}$ & $0.20 \pm 0.01^{\mathrm{b}}$ & $0.24 \pm 0.00^{\mathrm{a}}$ & $0.15 \pm 0.01^{\mathrm{c}}$ \\
\hline Total carbohydrate & $35.33 \pm 0.13^{\mathrm{b}}$ & $35.47 \pm 0.03^{\mathrm{b}}$ & $35.14 \pm 0.06^{\mathrm{c}}$ & $34.64 \pm 0.06^{\mathrm{d}}$ & $36.33 \pm 0.06^{\mathrm{a}}$ \\
\hline \multirow{3}{*}{$\begin{array}{l}\text { Calorific value } \\
\text { (k cal/100 g sample) }\end{array}$} & $145.27 \pm 0.68^{\mathrm{b}}$ & $146.13 \pm 0.46^{\mathrm{b}}$ & $143.62 \pm 0.30^{\mathrm{c}}$ & $142.23 \pm 0.40^{\mathrm{d}}$ & $148.89 \pm 0.05^{\mathrm{a}}$ \\
\hline & \multicolumn{4}{|c|}{ Heat Processed Sample Garri (eba) in Locations } & \\
\hline & Eleme & Ogoni & Okrika & Nembe & Abakaliki \\
\hline Moisture content & $66.70 \pm 0.02^{\mathrm{b}}$ & $66.43 \pm 0.13^{\mathrm{c}}$ & $66.93 \pm 0.03^{\mathrm{a}}$ & $65.90 \pm 0.02^{\mathrm{e}}$ & $66.02 \pm 0.03^{\mathrm{d}}$ \\
\hline Dry matter & $33.30 \pm 0.02^{\mathrm{b}}$ & $33.57 \pm 0.13^{\mathrm{c}}$ & $33.07 \pm 0.03^{\mathrm{d}}$ & $34.10 \pm 0.02^{\mathrm{c}}$ & $33.98 \pm 0.03^{\mathrm{a}}$ \\
\hline Crude protein & $0.35 \pm 0.04^{\mathrm{a}}$ & $0.34 \pm 0.01^{\mathrm{ab}}$ & $0.31 \pm 0.00^{\mathrm{b}}$ & $0.33 \pm 0.00^{\mathrm{ab}}$ & $0.35 \pm 0.00^{\mathrm{a}}$ \\
\hline Ash & $1.90 \pm 0.10^{\mathrm{b}}$ & $2.45 \pm 0.15^{\mathrm{a}}$ & $1.55 \pm 0.05^{\mathrm{c}}$ & $1.20 \pm 0.00^{\mathrm{d}}$ & $1.40 \pm 0.10^{\mathrm{c}}$ \\
\hline Crude fat & $0.11 \pm 0.00^{\mathrm{b}}$ & $0.12 \pm 0.01^{\mathrm{a}}$ & $0.10 \pm 0.00^{\mathrm{c}}$ & $0.11 \pm 0.00^{\mathrm{b}}$ & $0.10 \pm 0.00^{c}$ \\
\hline Total carbohydrate & $30.94 \pm 0.07^{\mathrm{c}}$ & $30.66 \pm 0.25^{\mathrm{d}}$ & $31.12 \pm 0.04^{\mathrm{c}}$ & $32.46 \pm 0.02^{\mathrm{a}}$ & $32.13 \pm 0.13^{\mathrm{b}}$ \\
\hline $\begin{array}{l}\text { Calorific value } \\
\text { (k cal/100 g sample) }\end{array}$ & $126.15 \pm 0.45^{\mathrm{b}}$ & $125.04 \pm 1.05^{\mathrm{c}}$ & $126.59 \pm 0.15^{\mathrm{b}}$ & $132.15 \pm 0.08^{\mathrm{a}}$ & $131.29 \pm 0.38^{\mathrm{a}}$ \\
\hline
\end{tabular}

*Values are means tstandard deviations of triplicate determinations. Values in the same row (for raw and for heat processed) bearing the same superscript letters are not significantly different at $5 \%$ level.

Crude protein was low in yam tuber from all the studied areas. The highest protein level of $1.71 \pm 0.06 \%$ was obtained from Abakaliki followed by Eleme $(1.57 \pm 0.23 \%)$ and was lowest in Okrika $(1.39 \pm 0.13 \%)$. Ash ranged from $0.97 \pm 0.06 \%$ in Abakaliki to $2.57 \pm 0.12 \%$ in Okrika. Crude fat was low and ranged from $0.10 \pm 0.00 \%$ in Okrika to $0.17 \pm 0.01 \%$ in Nembe. In all the areas investigated, total carbohydrate was high and ranged from $33.32 \pm 0.42 \%$ in
Ogoni to $35.21 \pm 0.13 \%$ in Abakaliki, while the calorific value in kcal per $100 \mathrm{~g}$ sample was low. Highest value of 149.08 was obtained from Abakaliki followed by Eleme (142.55) with the lowest value of 140.09 in Ogoni.

The results of the proximate composition of raw and heat processed cassava garri (eba) are presented in Table 2, while those of raw and heat processed cassava (fufu) are embodied in Table 3. 
Table 3 Proximate Composition (\%) of Raw and Heat Processed Cassava from Oil Producing Areas (Eleme, Ogoni, Okrika, Nembe) and Abakaliki

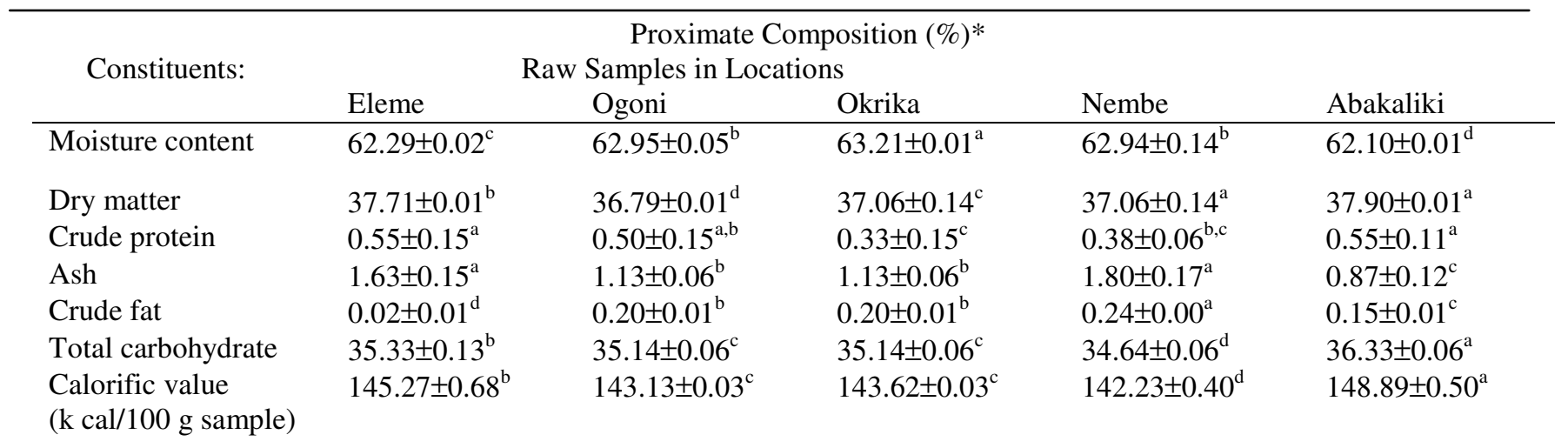

Heat Processed Samples (fufu) in Location.

\begin{tabular}{llllll} 
& Eleme & Ogoni & Okrika & Nembe & Abakaliki \\
\hline Moisture content & $64.69 \pm 0.01^{\mathrm{e}}$ & $65.78 \pm 0.10^{\mathrm{d}}$ & $68.79 \pm 0.06^{\mathrm{b}}$ & $69.49 \pm 0.24^{\mathrm{a}}$ & $68.45 \pm 0.06^{\mathrm{c}}$ \\
Dry matter & $35.31 \pm 0.01^{\mathrm{a}}$ & $34.22 \pm 0.10^{\mathrm{b}}$ & $31.21 \pm 0.06^{\mathrm{d}}$ & $30.51 \pm 0.24^{\mathrm{e}}$ & $31.55 \pm 0.06^{\mathrm{c}}$ \\
Crude protein & $0.33 \pm 0.11^{\mathrm{a}}$ & $0.32 \pm 0.01^{\mathrm{a}}$ & $0.27 \pm 0.05^{\mathrm{a}}$ & $0.31 \pm 0.02^{\mathrm{a}}$ & $0.33 \pm 0.11^{\mathrm{a}}$ \\
Ash & $1.00 \pm 0.00^{\mathrm{a}}$ & $0.75 \pm 0.05^{\mathrm{c}}$ & $0.90 \pm 0.00^{\mathrm{b}}$ & $1.05 \pm 0.05^{\mathrm{a}}$ & $0.90 \pm 0.00^{\mathrm{b}}$ \\
Crude fat & $0.10 \pm 0.00^{\mathrm{a}}$ & $0.05 \pm 0.00^{\mathrm{c}}$ & $0.10 \pm 0.00^{\mathrm{a}}$ & $0.08 \pm 0.01^{\mathrm{b}}$ & $0.05 \pm 0.00^{\mathrm{c}}$ \\
Total carbohydrate & $33.89 \pm 0.10^{\mathrm{a}}$ & $33.10 \pm 0.06^{\mathrm{b}}$ & $29.93 \pm 0.03^{\mathrm{d}}$ & $29.08 \pm 0.31^{\mathrm{e}}$ & $30.27 \pm 0.16^{\mathrm{c}}$ \\
Calorific value & $137.75 \pm 0.05^{\mathrm{a}}$ & $134.13 \pm 0.26^{\mathrm{b}}$ & $121.73 \pm 0.23^{\mathrm{d}}$ & $118.25 \pm 1.08^{\mathrm{e}}$ & $122.84 \pm 0.23^{\mathrm{c}}$ \\
(k cal/100 g sample) & & & & & \\
\hline
\end{tabular}

*Values are means tstandard deviations of triplicate determinations. Values in the same row (for raw and for heat processed) bearing the same superscript letters are not significantly different at $5 \%$ level.

Results in Tables 2 and 3 followed similar trend. For raw samples in Table 2 for instance, moisture content was high and ranged from $62.10 \pm 0.01 \%$ in Abakaliki to $63.21 \pm 0.01 \%$ in Okrika while crude protein was generally low with the highest value of $0.55 \pm 0.11 \%$ from Abakaliki and the lowest value of $0.33 \pm 0.15 \%$ from Okrika. Ash was highest in Nembe $(1.80 \pm 07 \%)$ followed by Eleme $(1.63 \pm 0.15 \%)$ and lowest in Abakaliki $(0.87 \pm 0.12 \%)$, while crude fat was low in samples from all the studied areas. Values from oil-producing areas which ranged from $0.20 \pm 0.01 \%$ in Eleme and Okrika to $0.25 \pm 0.01 \%$ in Ogoni were significantly $(\mathrm{p}<0.05)$ higher than $0.15 \pm 0.0 .01 \%$ obtained from Abakaliki (control). Total carbohydrate was highest in Abakaliki (36.33 $\pm 0.06 \%)$ followed by Eleme $(35.33 \pm 0.13 \%)$ and lowest in Nembe $(34.64 \pm 0.06 \%)$, while the energy values (kcal/100 g samples) ranged from 142.23 in Nembe to 148.89 in Abakaliki.
The proximate composition and energy values of raw and heat processed cocoyam tubers from different locations investigated are shown in Table 4. Values of moisture were also high in all the areas. The highest value of $68.05 \pm 0.04 \%$ was obtained from Ogoni followed by Nembe and Abakaliki where values did not differ significantly $(\mathrm{p}<0.05)$ and lowest in Eleme $(65.61 \pm 0.15 \%)$. Crude protein values were low across the studied areas and ranged from $1.39 \pm 0.46 \%$ in Nembe to $1.75 \pm 0.22 \%$ in Abakaliki. Ash which ranged from $2.03 \pm 0.06 \%$ to $2.57 \pm 0.12 \%$ in oil-producing areas was significantly higher $(\mathrm{p}<0.05)$ than value obtained in samples from Abakaliki (0.93 $\pm 0.06 \%)$. Crude fat was low in all the areas and ranged from $0.10 \pm 0.00 \%$ in Eleme to $0.15 \pm 0.01 \%$ in Nembe. Total carbohydrate ranged from $29.90 \pm 0.19 \%$ in Nembe to $30.45 \pm 0.19 \%$ in Abakaliki while the energy value in $\mathrm{kcal} / 100 \mathrm{~g}$ sample was highest in Abakaliki (139.92) followed by Eleme (129.65) 
Table 4: Proximate Composition (\%) of Raw and Heat Processed Cocoyam Tubers from Oil Producing Areas (Eleme, Ogoni, Okrika, Nembe) and Abakaliki

\begin{tabular}{|c|c|c|c|c|c|}
\hline \multirow[t]{2}{*}{ Constituents: } & \multicolumn{3}{|c|}{$\begin{array}{l}\text { Proximate Composition }(\%) \\
\text { Raw Samples in Locations }\end{array}$} & \multirow[b]{2}{*}{ Nembe } & \multirow[b]{2}{*}{ Abakaliki } \\
\hline & Eleme & Ogoni & Okrika & & \\
\hline Moisture content & $65.61 \pm 0.15^{\mathrm{c}}$ & $68.05 \pm 0.04^{\mathrm{a}}$ & $65.67 \pm 0.05^{\mathrm{c}}$ & $66.53 \pm 0.23^{\mathrm{b}}$ & $66.73 \pm 0.08^{b}$ \\
\hline Dry matter & $34.39 \pm 0.15^{\mathrm{a}}$ & $31.95 \pm 0.04^{\mathrm{c}}$ & $34.33 \pm 0.05^{\mathrm{a}}$ & $33.47 \pm 0.23^{\mathrm{b}}$ & $33.27 \pm 0.22^{\mathrm{b}}$ \\
\hline Crude protein & $1.42 \pm 0.33^{\mathrm{a}}$ & $1.46 \pm 0.33^{\mathrm{a}}$ & $1.42 \pm 0.11^{\mathrm{a}}$ & $1.39 \pm 0.46^{\mathrm{a}}$ & $1.75 \pm 0.22^{\mathrm{a}}$ \\
\hline Ash & $2.10 \pm 0.20^{\mathrm{b}}$ & $1.00 \pm 0.10^{\mathrm{c}}$ & $2.57 \pm 0.12^{\mathrm{a}}$ & $2.03 \pm 0.06^{\mathrm{b}}$ & $0.93 \pm 0.06^{\mathrm{c}}$ \\
\hline Crude fat & $0.10 \pm 0.00^{c}$ & $0.11 \pm 0.01^{\mathrm{b}}$ & $0.11 \pm 0.01^{\mathrm{b}, \mathrm{c}}$ & $0.15 \pm 0.01^{\mathrm{a}}$ & $0.11 \pm 0.01^{\mathrm{b}}$ \\
\hline Total carbohydrate & $30.77 \pm 0.63^{\mathrm{a}}$ & $29.38 \pm 0.36^{\mathrm{c}}$ & $30.23 \pm 0.25^{\mathrm{a}, \mathrm{b}}$ & $29.90 \pm 0.60^{\mathrm{b}, \mathrm{c}}$ & $30.48 \pm 0.19^{\mathrm{a}, \mathrm{b}}$ \\
\hline \multirow{3}{*}{$\begin{array}{l}\text { Calorific value } \\
\text { (k cal/100 g sample) }\end{array}$} & $129.65 \pm 1.36^{\mathrm{a}}$ & $124.38 \pm 0.68^{\mathrm{b}}$ & $127.60 \pm 0.68^{\mathrm{b}}$ & $126.48 \pm 1.11^{\mathrm{b}}$ & $129.92 \pm 0.53^{\mathrm{a}}$ \\
\hline & \multicolumn{5}{|c|}{ Heat Processed Samples in Locations } \\
\hline & Eleme & Ogoni & Okrika & Nembe & Abakaliki \\
\hline Moisture content & $68.07 \pm 0.05^{\mathrm{c}}$ & $70.03 \pm 0.05^{\mathrm{a}}$ & $68.03 \pm 0.06^{\mathrm{c}}$ & $68.17 \pm 0.02^{\mathrm{b}}$ & $68.01 \pm 0.01^{\mathrm{c}}$ \\
\hline Dry matter & $31.93 \pm 0.05^{\mathrm{a}}$ & $29.97 \pm 0.06^{\mathrm{c}}$ & $31.97 \pm 0.06^{\mathrm{a}}$ & $31.83 \pm 0.02^{\mathrm{b}}$ & $31.99 \pm 0.01^{\mathrm{a}}$ \\
\hline Crude protein & $0.77 \pm 0.33^{\mathrm{b}}$ & $1.25 \pm 0.22^{\mathrm{a}}$ & $0.98 \pm 0.11^{\mathrm{a}, \mathrm{b}}$ & $1.06 \pm 0.22^{\mathrm{a}, \mathrm{b}}$ & $1.09 \pm 0.00^{\mathrm{a}, \mathrm{b}}$ \\
\hline Ash & $1.85 \pm 0.05^{\mathrm{a}}$ & $0.70 \pm 0.10^{\mathrm{c}}$ & $1.20 \pm 0.00^{\mathrm{b}}$ & $1.65 \pm 0.05^{\mathrm{a}, \mathrm{b}}$ & $1.20 \pm 0.06^{\mathrm{b}}$ \\
\hline Crude fat & $0.08 \pm 0.01^{\mathrm{d}}$ & $0.10 \pm 0.00^{c}$ & $0.10 \pm 0.00^{c}$ & $0.12 \pm 0.00^{\mathrm{b}}$ & $0.80 \pm 0.00^{\mathrm{a}}$ \\
\hline Total carbohydrate & $29.24 \pm 0.23^{\mathrm{a}, \mathrm{b}}$ & $27.93 \pm 0.04^{c}$ & $29.68 \pm 0.16^{\mathrm{a}}$ & $29.00 \pm 0.30^{\mathrm{b}}$ & $28.90 \pm 0.59^{\mathrm{b}}$ \\
\hline $\begin{array}{l}\text { Calorific value } \\
\text { (k cal/100 g sample) }\end{array}$ & $120.71 \pm 0.35^{\mathrm{c}}$ & $117.52 \pm 0.24^{\mathrm{d}}$ & $123.57 \pm 0.23^{\mathrm{b}}$ & $121.31 \pm 0.28^{\mathrm{c}}$ & $127.17 \pm 2.36^{\mathrm{a}}$ \\
\hline
\end{tabular}

* Values are means tstandard deviations of triplicate determinations. Values in the same row (for raw and for heat processed) bearing the same superscript letters are not significantly different at $5 \%$ level.

Table 5:Proximate Composition (\%) of Raw and Heat Processed Maize Samples from Oil Producing Areas (Eleme, Ogoni, Okrika, Nembe) and Abakaliki

\begin{tabular}{|c|c|c|c|c|c|}
\hline \multirow[t]{2}{*}{ Constituents: } & \multicolumn{4}{|c|}{ Proximate Composition (\%)* } & \multirow[b]{2}{*}{ Abakaliki } \\
\hline & Eleme & Ogoni & Okrika & Nembe & \\
\hline Moisture content & $55.19 \pm 0.01^{\mathrm{d}}$ & $57.41 \pm 0.01^{\mathrm{b}}$ & $56.25 \pm 0.12^{\mathrm{c}}$ & $59.26 \pm 0.02^{\mathrm{a}}$ & $51.14 \pm 0.04^{\mathrm{e}}$ \\
\hline Dry matter & $44.81 \pm 0.01^{\mathrm{b}}$ & $42.59 \pm 0.01^{\mathrm{d}}$ & $43.75 \pm 0.12^{\mathrm{c}}$ & $40.74 \pm 0.02^{\mathrm{e}}$ & $48.86 \pm 0.04^{\mathrm{a}}$ \\
\hline Crude protein & $8.75 \pm 0.00^{\mathrm{b}, \mathrm{c}}$ & $9.48 \pm 1.26^{\mathrm{b}}$ & $9.30 \pm 0.54^{\mathrm{b}}$ & $8.02 \pm 0.63^{\mathrm{c}}$ & $10.44 \pm 0.00^{\mathrm{a}}$ \\
\hline Ash & $3.03 \pm 0.15^{\mathrm{a}}$ & $1.77 \pm 0.12^{\mathrm{d}}$ & $2.23 \pm 0.06^{\mathrm{c}}$ & $2.67 \pm 0.06^{\mathrm{b}}$ & $1.63 \pm 0.06^{\mathrm{d}}$ \\
\hline Crude fat & $4.40 \pm .20^{\mathrm{c}}$ & $5.74 \pm 0.00^{\mathrm{b}}$ & $3.15 \pm 0.15^{\mathrm{d}}$ & $4.25 \pm 0.05^{\mathrm{c}}$ & $6.79 \pm 0.14^{\mathrm{a}}$ \\
\hline Total carbohydrate & $28.62 \pm 0.14^{\mathrm{b}}$ & $25.60 \pm 1.22^{\mathrm{c}}$ & $29.06 \pm 0.25^{\mathrm{b}}$ & $25.08 \pm 0.63^{c}$ & $32.06 \pm 0.05^{\mathrm{a}}$ \\
\hline \multirow{2}{*}{$\begin{array}{l}\text { Calorific value }(\mathrm{K} \\
\mathrm{cal} / 100 \mathrm{~g} \text { sample }\end{array}$} & $189.09 \pm 0.70^{\mathrm{c}}$ & $191.99 \pm 0.46^{\mathrm{b}}$ & $181.77 \pm 0.24^{\mathrm{d}}$ & $173.54 \pm 0.54^{\mathrm{e}}$ & $210.05 \pm 0.31^{\mathrm{a}}$ \\
\hline & Eleme & $\begin{array}{l}\text { Heat Processe } \\
\text { Ogoni }\end{array}$ & $\begin{array}{l}\text { nples in Locations. } \\
\text { Okrika }\end{array}$ & Nembe & Abakaliki \\
\hline Moisture content & $64.61 \pm 0.01^{\mathrm{d}}$ & $65.69 \pm 0.15^{\mathrm{c}}$ & $68.41 \pm 0.09^{\mathrm{b}}$ & $68.83 \pm 0.05^{\mathrm{a}}$ & $64.43 \pm 0.12^{\mathrm{e}}$ \\
\hline Dry matter & $35.39 \pm 0.01^{\mathrm{a}}$ & $34.31 \pm 0.15^{\mathrm{b}}$ & $31.59 \pm 0.09^{c}$ & $31.17 \pm 0.05^{\mathrm{d}}$ & $35.57 \pm 0.12^{\mathrm{a}}$ \\
\hline Crude protein & $7.66 \pm 1.09^{\mathrm{a}}$ & $8.31 \pm 0.44^{\mathrm{a}}$ & $7.11 \pm 0.54^{\mathrm{a}}$ & $7.66 \pm 1.09^{\mathrm{a}}$ & $8.20 \pm 0.54^{\mathrm{a}}$ \\
\hline Ash & $2.70 \pm 0.01^{\mathrm{a}}$ & $1.08 \pm 0.05^{\mathrm{d}}$ & $1.95 \pm 0.05^{\mathrm{b}}$ & $1.80 \pm 0.00^{\mathrm{c}}$ & $1.10 \pm 0.00^{\mathrm{d}}$ \\
\hline Crude fat & $2.35 \pm 0.01^{\mathrm{e}}$ & $3.20 \pm 0.00^{c}$ & $3.02 \pm 0.01^{\mathrm{d}}$ & $3.91 \pm 0.01^{\mathrm{b}}$ & $5.46 \pm 0.01^{\mathrm{a}}$ \\
\hline Total carbohydrate & $22.69 \pm 0.98^{\mathrm{a}}$ & $21.75 \pm 0.27^{\mathrm{a}}$ & $19.51 \pm 0.69^{b}$ & $17.81 \pm 1.14^{\mathrm{c}}$ & $23.07 \pm 0.64^{\mathrm{a}}$ \\
\hline $\begin{array}{l}\text { Calorific value } \\
\text { (k cal/100 g sample) }\end{array}$ & $142.50 \pm 0.42^{\mathrm{c}}$ & $149.05 \pm 0.78^{b}$ & $133.65 \pm 0.51^{\mathrm{e}}$ & $137.04 \pm 0.14^{\mathrm{d}}$ & $153.39 \pm 0.47^{\mathrm{a}}$ \\
\hline
\end{tabular}


and lowest in Ogoni (124.38). The proximate composition of raw and heat processed maize from the locations studied are presented in Table 5. Moisture contents of raw samples from oilproducing areas which ranged from $55.19 \pm 0.01$ to $59.26 \pm 0.02 \%$ were significantly $(\mathrm{p}<0.05)$ higher than that of Abakaliki which was $51.14 \pm 0.04 \%$. Crude protein was highest in samples from Abakaliki (10.44 $\pm 0.00 \%)$ followed by Ogoni $(9.48 \pm 0.12 \%)$ and lowest in samples from Nembe $(8.02 \pm 0.63 \%)$ Ash was significantly higher in oil-producing areas compared with Abakaliki. Crude fat was highest in samples from Abakaliki followed by Ogoni and lowest in Okrika (Table 5). Total carbohydrate ranged from $25.08 \pm 0.63 \%$ in Nembe to $32.06 \pm 0.05 \%$ in Abakaliki while calorific value also ranged from 173.54 $\mathrm{kcal} / 100 \mathrm{~g}$ in Nembe to $210.05 \mathrm{kcal} / 100 \mathrm{~g}$ in Abakaliki.

It is discernible from Tables 1-5 that the heat processing method employed increased moisture content of the staple foods evaluated in all the areas investigated. However, cooking generally decreased the dry matter, crude protein, ash, crude fat, total carbohydrate and the energy value of all the samples analysed.

\section{DISCUSSION}

From the data shown in Tables 1-5, it can be seen that moisture content in all the samples from oil-producing areas and also the control area of Abakaliki were quite high. The high moisture levels suggest that the staple foods analysed may not store for a long time due to high water activity. This may cause quality problem as the samples may be liable to bacterial spoilage during storage ${ }^{12,13}$. Crude protein content of the staples from Abakaliki were generally higher compared with those from oil-producing areas due probably to favourable soil fertility and environmental condition. It has been reported that Abakaliki soils were high in nitrate $^{14}$.

In his work with Puccinellia maritama, Amakiri ${ }^{15}$ showed that plant cell membranes are disrupted by crude oil pollution and thus cell organelles such as ribosomes which are the sites of protein synthesis are affected in crude oil damaged cells. The low levels of plant proteins in all the samples analysed do not justify the use of yams, cassava, cocoyams and maize in diets as protein source for the alleviation of kwashiorkor arising from protein malnutrition. These samples cannot contribute to the daily protein requirement of $23-56 \mathrm{~g}$ for humans ${ }^{16}$. The low levels of protein in these samples would therefore require dietary supplementation with animal proteins or complementary proteins from legumes especially in diets meant for children and pregnant women.

The significantly $(\mathrm{p}<0.05)$ higher ash contents (Tables 1-5) of the staple food samples from oilproducing areas of the Niger Delta compared with the values from control samples may be attributable to crude oil pollution or contamination of the soils. The high ash contents of samples from oil-producing areas may suggest higher level of mineral elements in these samples as against values in food samples from non-oil contaminated environments. Since uptake of water, salts and mineral elements are carried out by plant roots; food crops grown on crude oil polluted farmlands may accumulate these minerals in their tissues. It has been demonstrated that some garden vegetables and root crops are capable of accumulating high levels of minerals from the soil ${ }^{17-19}$.

Crude fat was generally low in the samples irrespective of location except in maize grains where values were significantly higher than in any other staple food investigated. The low level of crude fats in all the samples analysed from oil-producing and control areas may be of benefit to individuals suffering from hyperlipidaemia because of the role of fat in potentiating one's risk of developing certain kinds of cancer and heart disease. The high levels of total carbohydrate in the staple foods indicate that they contribute significantly to the energy content of the food materials. The total carbohydrate contents of raw staple foods from oil-producing areas were in general significantly $(\mathrm{p}<0.05)$ lower than values from Abakaliki. These relatively lower levels of carbohydrate may be due to inhibition of photosynthetic process arising from interference with light and 
gas exchange caused by gas flaring in oilproducing environments ${ }^{15}$. There could also be interference with uptake of water and nutrients due to crude oil pollution, effluent discharge and waste disposal in farmlands ${ }^{15}$. Crude oil pollution has long been reported to reduce the rate of photosynthesis by flowering plants ${ }^{20}$. The relatively higher energy content of these raw staple foods from Abakaliki over values from oil-producing areas of the Niger Delta may be attributable to relatively higher nutrient (crude protein, crude fat and total carbohydrate) levels arising probably from better soil fertility and favourable climatic and environmental conditions. For yam tubers (Table 1) for instance, the consumption of up to $350 \mathrm{~g}$ per day per consumer of raw samples from Eleme, Ogoni, Okrika, Nembe and Abakaliki would provide energy values of 498.9, 490.3, 490.8, 493.8 and $521.8 \mathrm{kcal}$ respectively while for heat processed yam tubers at the same amount of intake, energy values would be $425.5,469.1$, $479.9,485.9$ and $462.6 \mathrm{kcal}$ respectively. At the same level of consumption per day for other raw and heat processed staple foods (Tables 2-5), similar values in energy content were obtained. The daily energy requirement of 2500-3000 kcal has been reported for adults ${ }^{21}$. Again, the $\mathrm{FAO}^{22}$ recommended range was $800-1200 \mathrm{kcal}$. It therefore follows that none of the raw and heat processed food materials investigated from oilproducing and control areas would meet the daily energy need of an adult if consumed at $350 \mathrm{~g}$ per day, and none would also meet the $\mathrm{FAO}^{22}$ recommended values. These samples, irrespective of source (oil-producing or non oilproducing area) cannot therefore be consumed as the sole source of energy to humans. The low level of energy could be attributable to very low levels of crude protein and crude fat as well as high levels of moisture in the samples. In order to derive full nutritional potential of these staple foods, they should be consumed together with protein-and fat-giving foods since low energy may lead to low birth weight and increased infant mortality ${ }^{23}$.

Cooking as a form of heat processing increased moisture content but decreased crude protein, ash, crude fat, total carbohydrate and calorific value in all the samples analyzed. The decrease may be due to loss of soluble beneficial nutrients (sugars and proteins) by leaching into the cooking solution that is usually discarded. The heat treatment may also have denatured the proteins rendering them more susceptible to proteolytic degradation. The decrease in the levels of these nutrients due to heat processing was not significant $(\mathrm{p}>0.05)$, and hence not considerable for food materials that must be heat treated in order to destroy natural antinutritional factors and derive their full nutritional potential.

Concluding, proximate composition of four staple foods (yam, cassava, cocoyam and maize) from oil-producing areas (Eleme, Ogoni, Okrika, Nembe) of the Niger Delta and the control area (Abakaliki) showed that the samples were high in moisture and carbohydrate but low in crude protein, ash, crude fat and energy content. Values of crude protein, total carbohydrate and energy content were generally lower in samples from oil-producing areas than samples from Abakaliki while the reverse was the case for ash content. In general and except for ash and moisture content, the level of nutrients in staple foods from oil-producing areas of the Niger Delta, Nigeria was significantly $(p<0.05)$ lower than values obtained in similar samples from non oil-producing area of Abakaliki in Ebonyi State, Nigeria. Heat treatment increased moisture but decreased crude protein, ash, crude fat, total carbohydrate and energy content in all the food samples irrespective of source.

\section{REFERENCES}

1. Okpokwasili, G. C. and Nwabuzor, C. N. (1988) Primary biodegradation of anionic surfactants in laundry detergents. Chemosphere 17:2175-2182

2. Dejong, E. (1980) The effect of crude oil spills on cereals. Environ. pollution 22:187-196.

3. Jones, M. T. and Stockinger, K. R. (1976) Effect of fertilizer on exchangeable cation content at different depths in cropped soils at two sites in the Nigerian Savanna. Trop. Agric. 53:243-254.

4. Adam, R. S. and Ellis, R. (1960) Some physical and chemical changes in soil 
brought about by saturation with natural gas. Soil Sci. Amer. Proc. 24:41-44.

5. Onyeike, E. N., Abbey, B. W. and Anosike, E. O. (1991) Kinetics of heat inactivation of trypsin inhibitors from the African yam bean (Sphenostylis stenocarpa).Food Chem 40:9-23

6. Eka, O. U. (1985) The chemical composition of yam tubers, in Advances in Yam Research: The Biochemistry and Technology of Yam Tubers, Vol. 1. ed by Osuji GO, published by Biochemical Society of Nigeria in collaboration with ASUTECH, Enugu, pp. 51-75.

7. Onwueme, I. C. (1978) The Tropical Tuber Crops. John Wiley and Sons, Chichester, London. p. 234

\section{Association of Official Analytical Chemists,} (1984) Official Methods of Analysis, $14^{\text {th }}$ edn, Arlington, VA, USA

9. Onyeike, E. N. and Onwuka, O. (1999) Chemical composition of some fermented vegetable seeds used as soup condiments in Nigeria East of the Niger. Global J. Pure Appl. Sci. 5:337-342

10. Onyeike, E. N. and Acheru, G. N. (2002) Chemical composition of selected Nigerian oil seeds and physicochemical properties of the oil extracts. Food Chem. 77:431-437

11. Duncan, D. B. (1955) Multiple range and multiple F tests. Biometrics 11:1-42.

12. Atkinson, I. S., Cecil, S. R., Woodroof, J. G. and Shelor, E. (1947) Extending keeping quality of pork meals. Food Ind. 19:1198-1199

13. Onyeike, E. N., Ihugba, A. C. and George, C. (2003) Influence of heat processing on the nutrient composition of vegetable leaves consumed in Nigeria. Plant Foods Hum. Nutr. 58:1-11

14. Ezeonu, F. C., Egboka, B. C. E. and Eze, S. S. (1994) Fertilizer application, nitrate loading patterns, water resources quality and their ecotoxicogical implications in Awka area of Nigeria. Environ. Toxic Water Quality 9:141-143.

15. Amakiri, J. O. (1981) Effect of Crude oil pollution on the germination and subsequent growth of Zea mays, Hibicus esculentus and Capsidium frustescens in Niger Delta. M.Sc Thesis, Department of Botany, University of Port Harcourt

16. National Research Council (1974) Recommended daily dietary allowance. Nutr. Rev. 31:373-395

17. Garcia, W. J., Blessing, C. W., Ingleth, G. E. and Kwolek, W. F. (1981) Metal accumulation and crop yield for a variety of edible crops grown in diverse soil media amended with sewage sludge. Environ Sci Tech 15:793-804

18. Khan, D. H. and Frankland, B. (1983) Effects of cadmium and lead on radish plants with particular reference to movements of metals through soil profile and plants. Plant and Soil 70:335-345

19. Cobb, G. P., Sand, S. K., Waters, M., Wixson, B. G. and Dorwars, K. E. (2000) Accumulation of heavy metals by vegetables grown in mine wastes. Environ Toxicol Chem 19: 600-607

20. Baker, J. M. (1970) Effects of Crude Oil on Plants. Environ Pollution 1:27-44

21. Bingham, S. (1978) Nutrition: A Consumer's Guide to Good Eating. Trans World, London, pp. 123-127

22. Food and Agriculture Organization (1972) Amino acid content of food and biological data on proteins, Report of FAO/United Nation Joint Committee, Rome, p116

23. Guthrie, H. A. and Picciano, M. F. (1995) Nutrition in Pregnancy, in Human Nutrition Mosby Year Book Inc, pp 303542 